\title{
A Rare Occurrence of Premature Birth and Recurrent Acute Pulmonary Oedema in the Mother due to Cushing's Syndrome: A Case Report
}

\author{
Dorothy Maria A/p Anthony Bernard, ${ }^{1}$ Ooi Xin Yi, ${ }^{1}$ Hema Lata A/p Veerasamy, ${ }^{1}$ \\ Mohamed Badrulnizam Long Bidin, ${ }^{2}$ See Chee Keong ${ }^{1}$ \\ ${ }^{1}$ Hospital Sultan Haji Ahmad Shah, Pahang, Malaysia \\ ${ }^{2}$ Hospital Kuala Lumpur, Kuala Lumpur, Malaysia
}

\begin{abstract}
Presentation of Cushing's syndrome during pregnancy is extremely rare. We report a 21-year-old female with Cushing's syndrome diagnosed at 23 weeks of gestation and had recurrent acute pulmonary oedema during the antepartum and postpartum period. She delivered prematurely via emergency caesarean section at 28 weeks of gestation. This case highlights the rare occurrence of recurrent acute pulmonary oedema during pregnancy and consequential premature birth in a patient with adrenal Cushing's. She was diagnosed with adrenal Cushing's during the postpartum period based on unsuppressed serum cortisol after overnight and low-dose dexamethasone suppression test with a suppressed ACTH. CT scan of the adrenal glands revealed a right adrenal cortical adenoma. The risk of complications in infants and mothers who suffer from Cushing's syndrome needs to be handled carefully. The diagnosis of Cushing's syndrome in pregnant women often overlaps and is difficult to establish in early pregnancy.
\end{abstract}

Key words: Cushing's syndrome, pregnancy, acute pulmonary oedema

\section{BACKGROUND}

Cushing's syndrome is rarely reported during pregnancy as most women with Cushing's syndrome usually present with subfertility secondary to hypercortisolism-induced ovulatory dysfunction. Only 220 cases of Cushing's syndrome in pregnancy have been reported in a recent systematic review over a 52-year period. ${ }^{1}$ The diagnosis of Cushing's syndrome in pregnancy is difficult and often missed as weight gain, development of striae, hyperglycemia and hypertension overlap with pregnancy itself. The levels of serum cortisol, corticosteroidbinding globulin and ACTH are increased during normal pregnancy and result in potential diagnostic difficulty. ${ }^{2}$ There are also no standardized serum cortisol and urinary cortisol reference ranges in pregnancy. ${ }^{3}$ In cases of successful conception, acute pulmonary oedema has been rarely reported. We report a patient with Cushing's syndrome diagnosed during pregnancy complicated by severe and life-threatening recurrent acute pulmonary oedema and premature birth.

\section{CASE}

A 21-year-old Malay female of middle socioeconomic status, first presented with acute pulmonary oedema at 23 weeks of gestation when she was admitted to the intensive care unit for four days for non-invasive ventilation. She had a BMI of $32.9 \mathrm{~kg} / \mathrm{m}^{2}$ with hypertension documented during pregnancy. Further assessment also revealed presence of gestational diabetes. She was investigated for Cushing's syndrome as she had facial acne, purplish abdominal striae, skin thinning and easy bruising (Figure 1). She had elevated 24-hour urinary cortisol and mildly elevated morning serum cortisol detected. However, her diagnosis of Cushing's syndrome was never confirmed as she defaulted her follow-up and was subsequently managed in multiple hospitals due to logistic reasons.

At 27 weeks of gestation, she was readmitted for acute pulmonary oedema (Figure 2 ) with hypertensive crisis. She required non-invasive ventilation during this admission and blood pressure was controlled with intravenous infusion of magnesium sulphate. A bedside echocardiogram showed an ejection fraction of $55 \%$ with presence of pericardial effusion at the base of the right atrium measuring $1.1 \mathrm{~cm}$ with presence of right atrial systolic collapse. In view of her unstable cardiac condition and hypertensive crisis, the collective decision between the obstetrician and cardiologist was to proceed with emergency caesarean section. A $1.1 \mathrm{~kg}$ baby girl was delivered prematurely at 27 weeks and 3 days period of gestation and was subsequently admitted to NICU for further care.

Following delivery, her condition improved with diuretics and blood pressure was controlled with 3 oral antihypertensive agents. Unfortunately, her hospitalization was prolonged due to dehiscence over her caesarean section surgical wound. Despite being discharged well after delivery, she was admitted for 2 further episodes of acute pulmonary oedema with hypertensive crisis, in which both episodes required ICU admission and ventilation. 

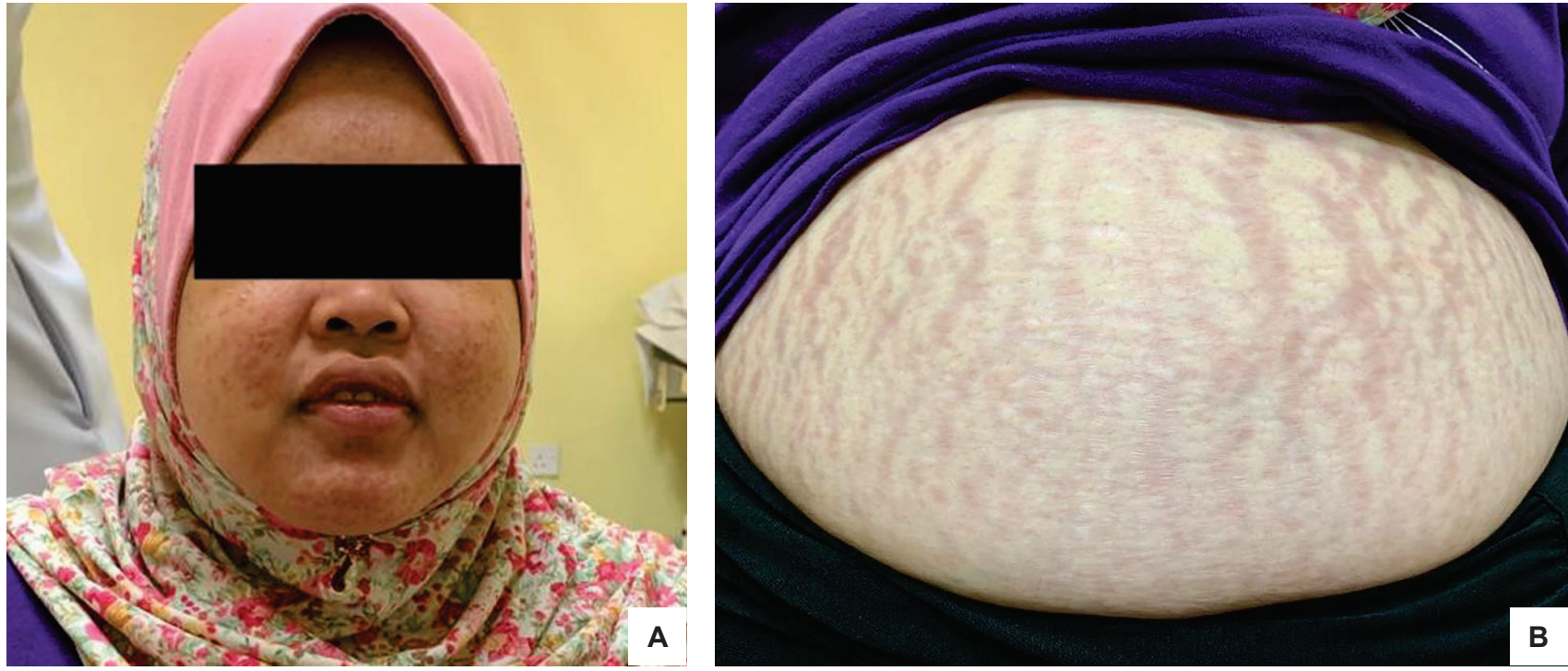

Figure 1. (A) Generalized acne on the patient's face; (B) Wide purplish striae on the patient's abdomen.

Table 1. Initial laboratory values

\begin{tabular}{ll}
\hline Laboratory & Patient values (Normal ranges) \\
\hline 24-hour urinary cortisol & $1712.4 \mathrm{nmol} / \mathrm{L}(53.2-876.3 \mathrm{nmol} / \mathrm{L})$ \\
8 am serum cortisol after 1mg over- & $646 \mathrm{nmol} / \mathrm{L}$ (unsuppressed) \\
night dexamethasone suppression & \\
$\begin{array}{l}\text { 8am serum cortisol after low-dose } \\
\text { dexamethasone suppression }\end{array}$ & $699 \mathrm{nmol} / \mathrm{L}$ (unsuppressed) \\
$\begin{array}{l}\text { Adrenocorticotrophic Hormone } \\
\text { (ACTH) }\end{array}$ & $1.1 \mathrm{pmol} / \mathrm{L}(<10.2 \mathrm{pmol} / \mathrm{L})$ \\
\hline Renal profile & \\
Blood urea nitrogen & $7.3 \mathrm{mmol} / \mathrm{L}(2.8-8.1 \mathrm{mmol} / \mathrm{L})$ \\
Serum sodium & $144 \mathrm{mmol} / \mathrm{L}(136-145 \mathrm{mmol} / \mathrm{L})$ \\
Serum potassium & $3.31 \mathrm{mmol} / \mathrm{L}(3.5-4.5 \mathrm{mmol} / \mathrm{L})$ \\
Serum chloride & $102 \mathrm{mmol} / \mathrm{L}(98-107 \mathrm{mmol} / \mathrm{L})$ \\
Serum creatinine & $38 \mathrm{mmol} / \mathrm{L}(62-106 \mathrm{mmol} / \mathrm{L})$ \\
\hline Liver function tests & \\
Total protein & $63 \mathrm{~g} / \mathrm{L}(66-87 \mathrm{~g} / \mathrm{L})$ \\
Albumin & $34 \mathrm{~g} / \mathrm{L}(35-52 \mathrm{~g} / \mathrm{L})$ \\
Total bilirubin & $9.1 \mathrm{umol} / \mathrm{L}(\leq 21 \mathrm{umol} / \mathrm{L})$ \\
Alanine transaminase & $62 \mathrm{U} / \mathrm{L}(\leq 33 \mathrm{U} / \mathrm{L})$ \\
Alkaline phosphatase & $148 \mathrm{U} / \mathrm{L}(35-104 \mathrm{U} / \mathrm{L})$ \\
\hline Complete blood count & \\
Hemoglobin & $12.3 \mathrm{~g} / \mathrm{dL}(11.5-17.0 \mathrm{~g} / \mathrm{dL})$ \\
Haematocrit & $35.5 \%(37.0-54.0 \%)$ \\
White blood cell count & $18.2 \times 10^{9} / \mathrm{L}\left(4.0-10.0 \times 10^{9} / \mathrm{L}\right)$ \\
Platelet count & $469 \times 10^{9} / \mathrm{L}\left(150-500 \times 10^{9} / \mathrm{L}\right)$ \\
\hline &
\end{tabular}

The first episode occurred two months after delivery, and again two weeks later. She received furosemide and BP lowering medication. Her diagnosis of ACTHindependent Cushing's Syndrome was only confirmed in between the two acute pulmonary oedema admissions during the postpartum period. She had unsuppressed serum cortisol after overnight and low-dose dexamethasone suppression test with suppressed ACTH (Table 1).

\section{Outcome and follow-up}

During her clinic review at 4 months postpartum, she complained of severe lower back pain which corresponded to T9 to L1 osteoporotic compression fracture (Figure 3). She required multiple analgesic medications to relieve her severe pain and she was given an immobilization brace. The pain resulted in her being home-bound and dependent on wheelchair for ambulation.
She also defaulted her follow-up during the postpartum period on several occasions resulting in a delay in her adrenal computerized tomography (CT) scan. Adrenal CT scan was only performed at 6 months postpartum which revealed a right medial limb adrenal adenoma measuring $3.1 \times 1.9 \times 2.9 \mathrm{~cm}$ with pre-contrast HU of 31 and $96 \%$ absolute contrast washout (Figure 4).

After much deliberation and counselling, she finally agreed to surgery. She received a short course of metyrapone preoperatively to control her cortisol and underwent an uncomplicated right open transabdominal adrenalectomy in July 2019. Intraoperative findings noted a right adrenal gland measuring $3 \times 3 \mathrm{~cm}$ in size. Histopathological examination of the right adrenal gland revealed a cortical adenoma.

During the postoperative period, she was started on oral hydrocortisone as cortisol replacement. Bisphosphonate (zoledronic acid) and cholecalciferol were also initiated for the severe osteoporosis. Her anti-hypertensive treatment was significantly reduced from 5 agents to only a single agent during her postoperative period.

\section{DISCUSSION}

The initial presentation for this case was unique as the patient presented with acute pulmonary oedema and hypertensive crisis during her pregnancy. The suspicion of Cushing's syndrome was strengthened with the presence of typical clinical features such as the purplish striae on the abdomen, facial acne, skin thinning and easy bruising. Her diagnosis was mainly delayed due to logistic issues and not due to difficulty of laboratory results interpretation. However, in the absence of hypertensive crisis and acute pulmonary oedema, the diagnosis of Cushing's syndrome may be missed due to some overlapping clinical features of Cushing's syndrome and normal pregnancy. Hence, the hunt for the diagnosis would require a high index of suspicion.

There is also a concern regarding the interpretation of screening and confirmatory tests for Cushing's syndrome 

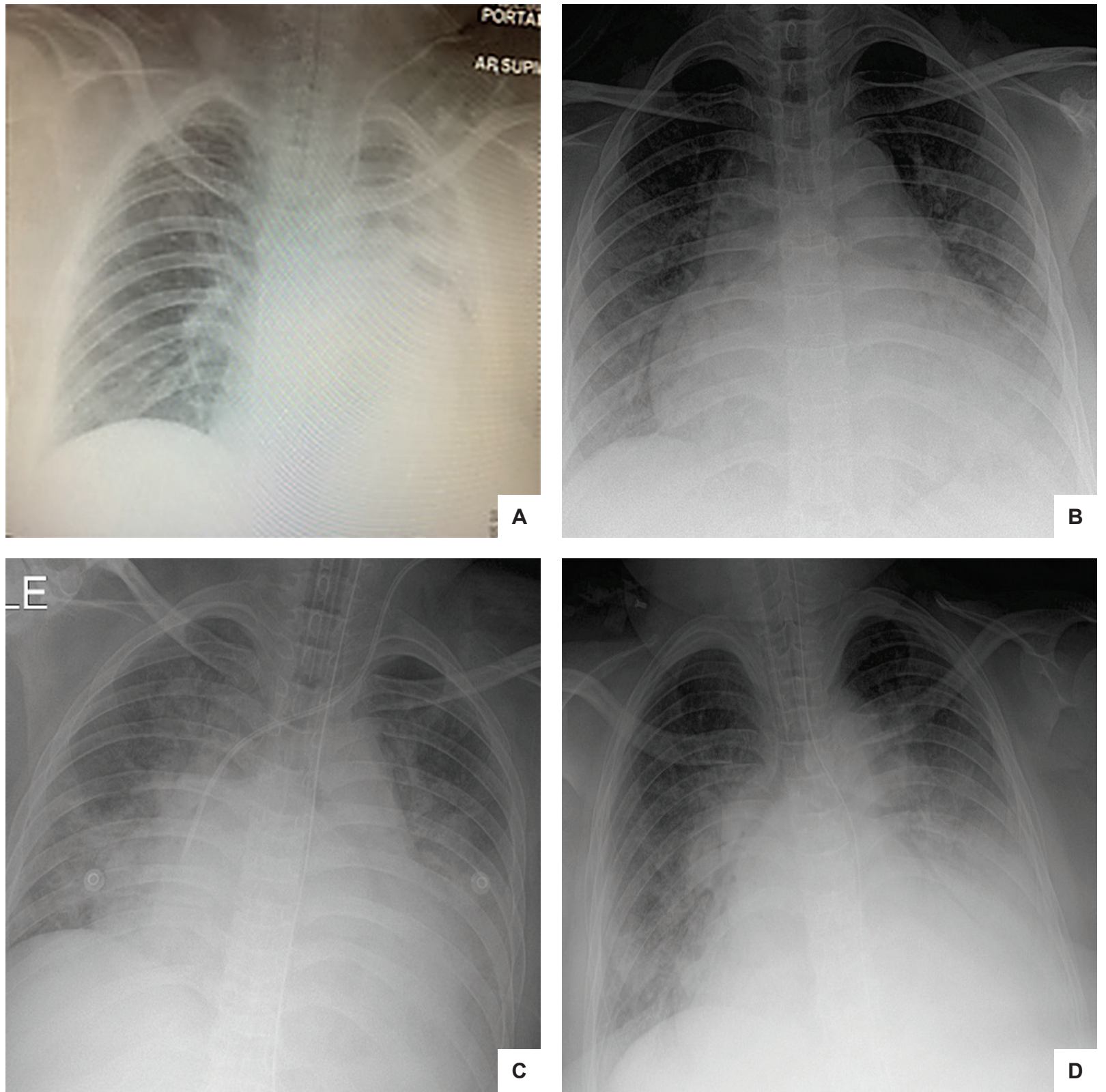

Figure 2. Serial chest radiographs showing acute pulmonary oedema from her previous 4 admissions. (A) left pleural effusion with bat's wings appearance and cardiomegaly; (B) left pleural effusion with cardiomegaly; (C) upper lobe diversion with bat's wings appearance and cardiomegaly; (D) left pleural effusion.

during pregnancy. There are physiological changes in cortisol regulation during the normal pregnancy. The rising estrogen level in pregnancy enhances the synthesis of cortisol-binding globulin in the liver. This in turn would increase the total plasma cortisol level during pregnancy. ${ }^{4}$ Plasma and urinary free cortisol are also increased during pregnancy due to the up-regulation of the hypothalamic-pituitary-adrenal axis. ${ }^{4}$ Hence, the morning cortisol may not be suppressed following an overnight dexamethasone suppression test, leading to a false positive result in normal pregnancy.

The high circulating cortisol in Cushing's syndrome during pregnancy may result in severe maternal and fetal complications. Maternal complications include increased risk of hypertension, diabetes and pre-eclampsia. Fetal complications include increased risk of intrauterine growth restriction and premature delivery. ${ }^{1}$ Osteoporosis is also a known complication of Cushing's syndrome like in our patient. Glucocorticoids cause loss of cortical osteocytes and lead to impaired bone healing which leads to osteoporosis. ${ }^{5}$

Heart failure has been previously reported as a presenting symptom of Cushing's syndrome. ${ }^{6}$ The occurrence of heart failure in a pregnant mother with Cushing's syndrome is rare and there are no reported cases of recurrent acute pulmonary oedema in pregnancy. Acute pulmonary oedema has only been previously reported once and it was attributed to pre-eclampsia. ${ }^{7}$ Kamenicky et al., has shown that excess steroid production in Cushing's syndrome resulted in decreased left ventricular stroke volume index, a lower ejection fraction and an increase in left ventricular mass compared to healthy controls. ${ }^{8}$ The causes of recurrent acute pulmonary oedema in this patient are multifactorial. The patient had poorly controlled hypertension due to poor compliance to antihypertensive 

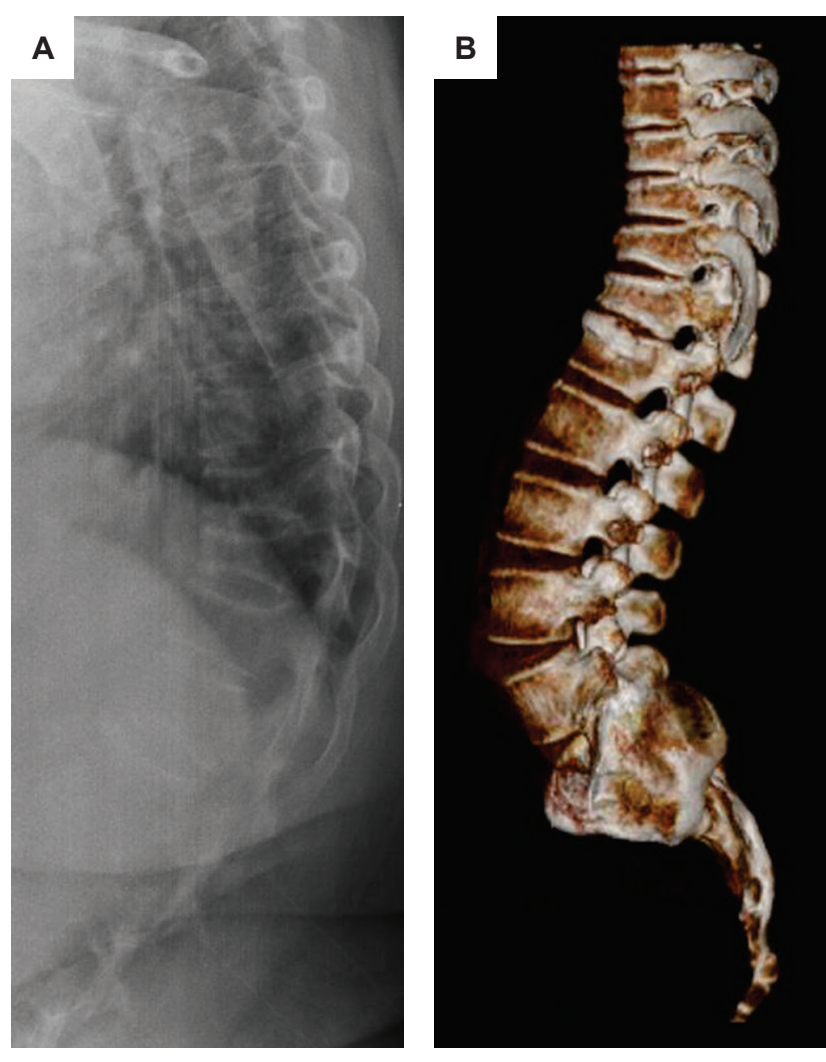

Figure 3. (A) Lateral view of thoracolumbar X-ray showing the compression fracture at T9 to L1 spine with osteopenic bone; (B) Volumetric 3D reconstruction of the thoracolumbar spine from CT images showing compression fracture.

medications and a persistently high circulating serum cortisol. Her management became extremely difficult as she frequently defaulted follow-up and had been seeking medical treatment from different centres. She was reviewed mainly in the acute setting during the acute pulmonary oedema admissions during her pregnancy.

During her admission for acute pulmonary oedema, echocardiogram assessment revealed presence of pericardial effusion and regional wall hypokinesia but her ejection fraction was mainly preserved. This observation is peculiar and would suggest possible association of Cushing's syndrome with diastolic dysfunction or heart failure with preserved ejection fraction. Prior study has also shown that patients with successful normalization of cortisol had improvement in their ejection fraction and reduction in left ventricle mass. ${ }^{8}$ In our patient, she had normal ejection fraction and had no regional wall abnormalities after her right adrenalectomy.

In our patient, her diagnosis of cortisol-producing right adrenal adenoma was only confirmed after delivery and imaging was done very much later. This is reflective of the cases of Cushing's syndrome in pregnancy reported in literature. Almost half of the cases of Cushing's syndrome in pregnancy were due to adrenal adenomas compared to only $15 \%$ in non-pregnant women. ${ }^{4}$ In our case, our management during pregnancy was centered on managing her acute pulmonary oedema, hypertensive crisis and determining her timing for delivery as she came for evaluation mainly in an emergency setting. Her acute pulmonary oedema management during pregnancy required treatment with intravenous diuretics and magnesium sulphate infusion.

In cases where Cushing's syndrome secondary to cortisolproducing adrenal adenoma was detected earlier in pregnancy without acute complications, medical and surgical treatment can be offered. Surgical unilateral adrenalectomy can safely be performed between 6 to 28 weeks of gestation. ${ }^{9}$ Medical therapy option in pregnancy is limited and indeed challenging. The use of metyrapone has been suggested but safety of medical therapy in pregnancy remains doubtful. ${ }^{1-3}$ Hence, management requires a multi-disciplinary discussion among the endocrinologist, endocrine surgeon and obstetrician to achieve management goals in pregnancy.

\section{CONCLUSION}

This case highlights the rare occurrence of recurrent acute pulmonary oedema and consequential premature birth
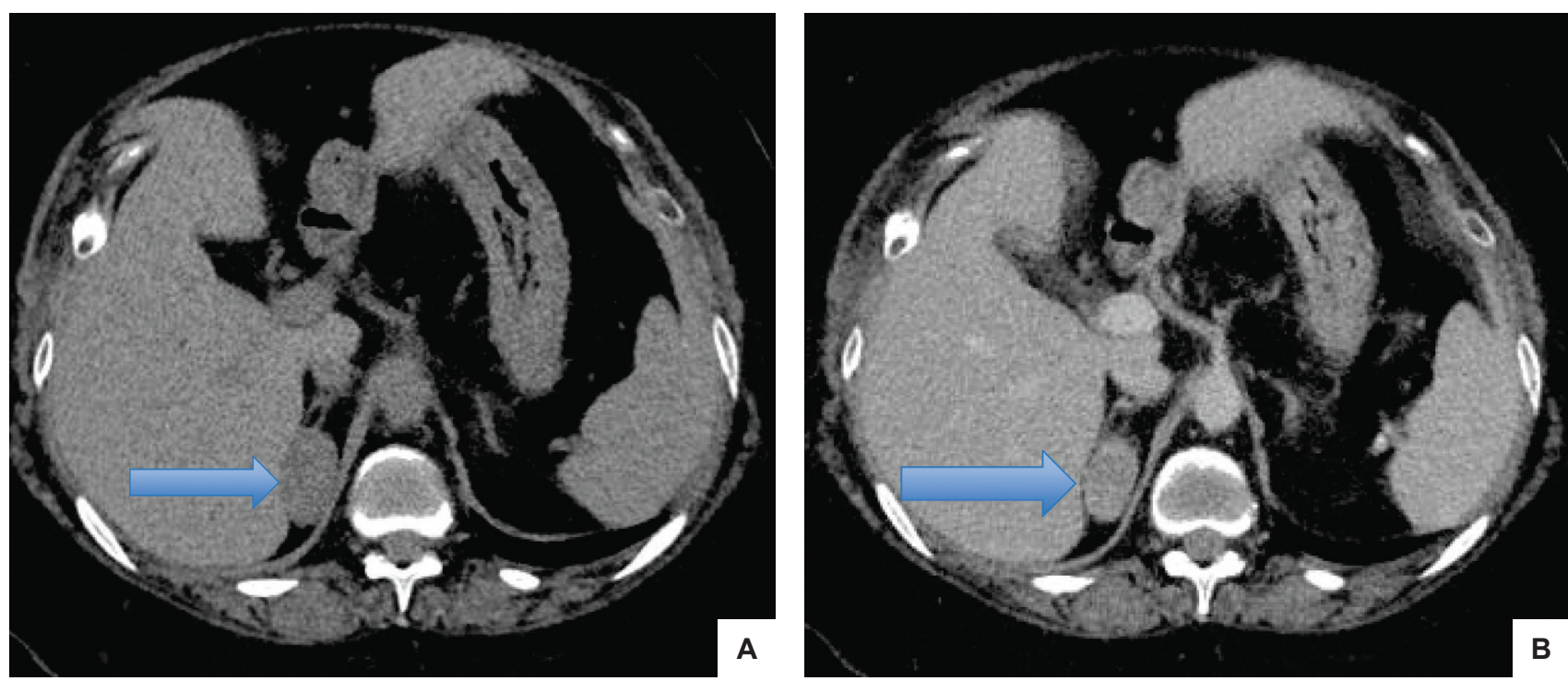

Figure 4. (A) CT adrenal pre-contrast scan showing the right adrenal adenoma (blue arrow); (B) CT adrenal post-contrast scan showing the well-defined hypodense lesion at the medial limb of the right adrenal gland. 
in a mother with Cushing's syndrome. Management of Cushing's syndrome in pregnancy is complicated and would definitely require a compliant patient and a coordinated endocrinologist-surgeon-obstetrician team.

\section{Ethical Consideration}

Patient consent was obtained before submission of the manuscript.

\section{Statement of Authorship}

All authors certified fulfilment of ICMJE authorship criteria.

\section{Author Disclosure}

The authors declared no conflict of interest.

\section{Funding Source}

None.

\section{References}

1. Caimari F, Valassi E, Garbayo P, et al. Cushing's syndrome and pregnancy outcomes: A systematic review of published cases. Endocrine. 2017;55(2):555-63. PMID: 27704478. https://doi.org/10.1007/ s12020-016-1117-0.

2. Machado MC, Fragoso MCBV, Bronstein MD. Pregnancy in patients with Cushing's syndrome. Endocrinol Metab Clin North Am. 2018; 47(2):441-9. PMID: 29754643. PMID: 29754643. https://doi.org/10.1016/j. ecl.2018.02.004
3. Brue T, Amodru V, Castinetti F. Management of endocrine diseases: Management of Cushing's syndrome during pregnancy: Solved and unsolved questions. Eur J Endocrinol. 2018;178(6):R259-66. PMID: 29523633. https://doi.org/10.1530/EJE-17-1058.

4. Lindsay JR, Nieman LK. The hypothalamic-pituitary-adrenal axis in pregnancy: Challenges in disease detection and treatment. Endocr Rev. 2005;26(6):775-99. PMID: 15827110. https://doi.org/10.1210/ er.2004-0025.

5. Arnaldi G, Angeli A, Atkinson AB, et al. Diagnosis and complications of Cushing's syndrome: A consensus statement. J Clin Endocrinol Metab. 2003;88(12):5593-602. https://doi.org/10.1210/jc.2003-030871.

6. Johnston $\mathrm{PC}$, Atkinson $\mathrm{AB}$, Moore MJ, et al. An unusual cause of reversible cardiomyopathy. Ulster Med J. 2012;81(3):134-5. PMID: 23620612. PMCID: PMC3632823.

7. Choi WJ, Jung TS, Paik WY. Cushing's syndrome in pregnancy with a severe maternal complication: A case report. J Obstet Gynaecol Res. 2011; 37(2):163-7. PMID: 21159041. https://doi.org/10.1111/j.14470756.2010.01339.x.

8. Kamenický P, Redheuil A, Roux C, et al. Cardiac structure and function in Cushing's syndrome: A cardiac magnetic resonance imaging study. J Clin Endocrinol Metab. 2014;99(11):E2144-53. PMID: 25093618. PMCID: PMC4223435. https://doi.org/10.1210/jc.2014-1783.

9. Andreescu CE, Alwani RA, Hofland J, et al. Adrenal Cushing's syndrome during pregnancy. Eur J Endocrinol. 2017:177(5): K13-20. PMID: 28819015. https;//doi.org/10.1530/EJE-17-0263.

Authors are required to accomplish, sign and submit scanned copies of the JAFES Author Form consisting of: (1) Authorship Certification, that authors contributed substantially to the work, that the manuscript has been read and approved by all authors, and that the requirements for authorship have been met by each author; (2) the Author Declaration, that the article represents original material that is not being considered for publication or has not been published or accepted for publication elsewhere, that the article does not infringe or violate any copyrights or intellectual property rights, and that no references have been made to predatory/suspected predatory journals; (3) the Author Contribution Disclosure, which lists the specific contributions of authors; (4) the Author Publishing Agreement which retains author copyright, grants publishing and distribution rights to JAFES, and allows JAFES to apply and enforce an Attribution-Non-Commercial Creative Commons user license; and (5) the Conversion to Visual Abstracts (*optional for original articles only) to improve dissemination to practitioners and lay readers Authors are also required to accomplish, sign, and submit the signed ICMJE form for Disclosure of Potential Conflicts of Interest. For original articles, authors are required to submit a scanned copy of the Ethics Review Approval of their research as well as registration in trial registries as appropriate. For manuscripts reporting data from studies involving animals, authors are required to submit a scanned copy of the Institutional Animal Care and Use Committee approval. For Case Reports or Series, and Images in Endocrinology, consent forms, are required for the publication of information about patients; otherwise, appropriate ethical clearance has been obtained from the institutional review board. Articles and any other material published in the JAFES represent the work of the author(s) and should not be construed to reflect the opinions of the Editors or the Publisher.

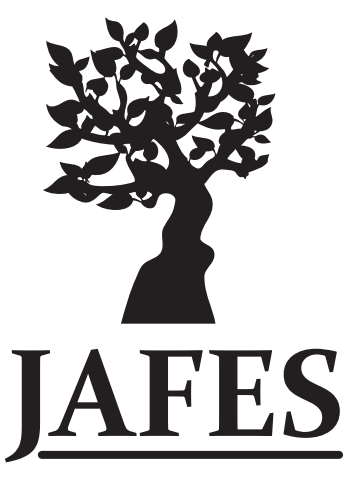

\section{Clinical controversies and disease updates are also welcome. Instructions to Authors available at www.ASEAN-endocrinejournal.org.}

